\title{
Inside the Hydrogen Atom
}

\author{
M. Nowakowski, N. G. Kelkar, D. Bedoya Fierro and A. D. Bermudez Manjarres \\ Departamento de Fisica, Universidad de los Andes \\ Cra. 1E, 18A-10, Bogotá, Colombia
}

\begin{abstract}
We apply the non-linear Euler-Heisenberg theory to calculate the electric field inside the hydrogen atom. We will demonstrate that the electric field calculated in the Euler-Heisenberg theory can be much smaller than the corresponding field emerging from the Maxwellian theory. In the hydrogen atom this happens only at very small distances. This effect reduces the large electric field inside the hydrogen atom calculated from the electromagnetic form-factors via the Maxwell equations. The energy content of the field is below the pair production threshold.
\end{abstract}

PACS numbers: 12.20.Ps, 13.40.Gp, 14.70.Bh, 31.15.aj 


\section{INTRODUCTION}

The electric field extracted from the electromagnetic form-factors of the proton is, in the short range (where it differs significantly from the Coulomb field), not a correction to the latter. This short range part is handled as a perturbation in calculating the energy correction which is possible due to the rapid fall-off of finite size contribution outside the proton. However, the magnitude of the electric field reaches a value up to $10^{3} \mathrm{MeV}^{2}$ which is not only extremely high, but also dangerously close to allowing electron-positron pair production. On the other hand, quantum corrections to Maxwell's equations known as the Euler-Heisenberg theory yield a different picture of the same physical phenomenon inside the hydrogen atom: the field becomes significantly smaller falling below the pair production threshold. We outline the theory which leads to this effect.

\section{THE SHORT RANGE ELECTRIC FIELD INSIDE THE HYDROGEN ATOM}

The electromagnetic properties of the nucleons are encoded in their two form-factors, $F_{1}$ and $F_{2}$. In calculating the elastic amplitude of the scattering process, $e p \rightarrow e p$, these form factors enter one of the vertices by making the replacement

$$
e \gamma_{\mu} \rightarrow e\left(F_{1}\left(q^{2}\right) \gamma_{\mu}+\frac{F_{2}\left(q^{2}\right)}{2 m_{p}} \sigma_{\mu \nu} q^{\nu}\right)
$$

The standard procedure to extract an interaction potential from the elastic amplitude is to (i) expand the latter in terms proportional to powers of $1 / c^{2}$ (which leads to the non-relativistic amplitude $\left.A_{N R} \equiv V(\mathbf{q})\right)$ and (ii) take the Fourier transform, i.e, $V(\mathbf{r})=$ $\int d^{3} q /(2 \pi)^{3} e^{i \mathbf{q} \cdot \mathbf{r}} V(\mathbf{q})$. In the case of the electromagnetic interaction, this leads to the Breit

equation [1, 2] and if we include the proton form-factors we explicitly take into account in the Breit equation the finite size of the proton in $V_{e p}(\mathbf{r})$ [3]. In terms of the so-called electric $\left(G_{E}\right)$ and magnetic $\left(G_{M}\right)$ Sachs form-factors $G_{E}=F_{1}+\frac{q^{2}}{4 m_{p}} F_{2}$ and $G_{M}=F_{1}+F_{2}$ the result of the scalar interaction potential in momentum space can be written as

$$
V_{e p}(\mathbf{q})=e\left[\frac{G_{E}(\mathbf{q})}{\mathbf{q}^{2}}-\frac{1}{8 m_{p}^{2}} G_{E}(\mathbf{q})-\frac{1}{8 m_{e}^{2}} G_{E}(\mathbf{q})\right] .
$$

In principle, the Breit equation gives also the fine and hyperfine structure of the interaction which have to be added to the term above. We do not spell it out explicitly here since we 


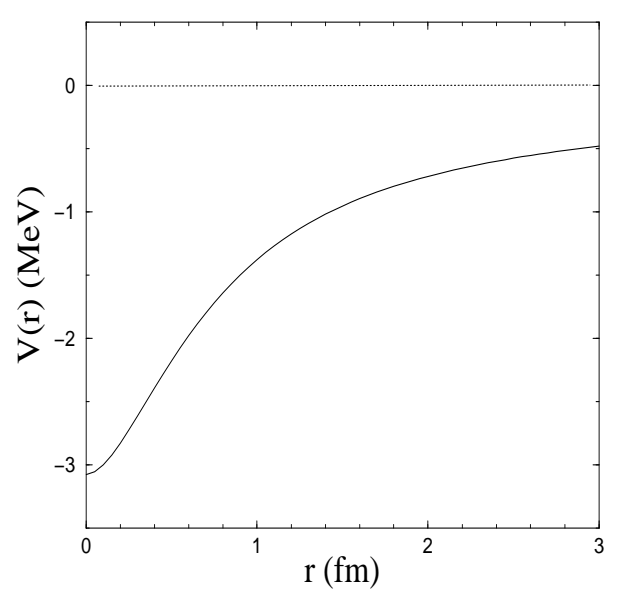

FIG. 1: The electric potential $V_{p}^{C}$.

will not make use of it. For details we refer to [4]. We can identify the first term as the Coulomb term (modified due to finite size) $V_{C}$, the second term is called the proton Darwin $V_{p D}$ and the third the electron Darwin term $V_{e D}$. We identify the electric potential of the proton from the Breit equation as terms which are (i) scalar (no spin and no momentum terms which appear in the fine and hyperfine part) and (ii) independent from the properties of the test particle. By this token the proton Darwin term can be part of the electric potential, but the electron Darwin not. This has some consequences for the electric field at small distances inside the proton and also for the charge distribution. Hence we write $V_{e p}(\mathbf{r})=e V_{C}(\mathbf{r})+V_{p D}(\mathbf{r})+V_{e D}(\mathbf{r})=e V_{p}(\mathbf{r})+V_{e D}(\mathbf{r})$ where $V_{p}$ is the proton electric potential. As an example let us take the dipole parametrization $G_{E}\left(\mathbf{q}^{2}\right)=1 /\left(1+\mathbf{q}^{2} / m^{2}\right)^{2}$. Then we obtain (see also figure 1)

$$
V_{p}^{C}=\frac{e}{r}\left(1-e^{-m r}\left(1+\frac{m r}{2}\right)\right)
$$

Since the main issues which we want to emphasize will remain mostly insensitive to the parametrization of the electric form-factors, we shall use the dipole parameterization where the Darwin terms can also be calculated analytically. The electric field $\mathbf{E}_{p}$ calculated from $V_{p}^{C}$ (which includes the form-factors) can be now as strong as $10^{3} \mathrm{MeV}^{2}$. It is reasonable to ask if this is indeed the case. We will give a partial answer in the following two sections starting with a discussion of the the non-linear Euler-Heisenberg theory. 


\section{STATIC ELECTRIC FIELD FROM EULER-HEISENBERG THEORY}

At the Born level, photons do not interact with each other. However, the box diagram with four external photon (light-light scattering) legs is an example of how quantum mechanics effectively introduces a four photon vertex. One can introduce this effect into the Lagrangian language which is known as the Euler-Heisenberg theory. In accordance with the gauge invariance we have to formulate the theory in terms of the two gauge invariant quantities

$$
\begin{aligned}
& \frac{1}{4} F_{\mu \nu} F^{\mu \nu}=-\frac{1}{2}\left(\mathbf{E}^{2}-\mathbf{B}^{2}\right), \\
& \frac{1}{4} F_{\mu \nu} \tilde{F}^{\mu \nu}=\mathbf{E} \cdot \mathbf{B} .
\end{aligned}
$$

Then, we can expect that in the first approximation (weak field approximation) the new part of the Lagrangian $\delta \mathcal{L}_{E H}$ is quadratic in these invariants. Indeed, Euler and Heisenberg obtained [5]

$$
\delta \mathcal{L}_{E H}=\eta\left(\left(\mathbf{E}^{2}-\mathbf{B}^{2}\right)^{2}+7(\mathbf{E} \cdot \mathbf{B})^{2}\right)
$$

with

$$
\eta=\frac{\alpha^{2}}{360 \pi^{2} m_{e}^{2}}
$$

Our requirement of weak fields means that in $\mathcal{L} \sim F^{2}\left(1+\eta F^{2}\right)(F \sim E, B)$ the new term must be small as compared to one or $\eta E^{2} \ll 1$ and $\eta B^{2} \ll 1$. It then follows that $E \ll 2 \times 10^{3} \mathrm{MeV}^{2}$. The new inhomogeneous Maxwell's equations now read:

$$
\begin{aligned}
\nabla \cdot \mathbf{D} & =\rho, \\
\nabla \times \mathbf{H}-\frac{\partial \mathbf{D}}{\partial t} & =\mathbf{j},
\end{aligned}
$$

with

$$
\begin{aligned}
& \mathbf{D} \equiv \mathbf{E}+4 \pi \mathbf{P} \\
& \mathbf{P}=\eta\left[4 \mathbf{E}\left(\mathbf{E}^{2}-\mathbf{B}^{2}\right)+14 \mathbf{B}(\mathbf{E} \cdot \mathbf{B})\right]
\end{aligned}
$$

and

$$
\begin{aligned}
\mathbf{H} & \equiv \mathbf{B}-4 \pi \mathbf{M}, \\
\mathbf{M} & =\eta\left[-4 \mathbf{B}\left(\mathbf{E}^{2}-\mathbf{B}^{2}\right)+14 \mathbf{E}(\mathbf{E} \cdot \mathbf{B})\right] .
\end{aligned}
$$


In the electrostatic case, putting $\mathbf{B}=0$ and neglecting all time derivatives we can write

$$
\nabla \cdot \mathbf{D}=\rho=\nabla \cdot \mathbf{E}_{0},
$$

where $\mathbf{E}_{0}$ is the electric field as calculated using the Maxwellian theory with $\rho$ (we treat the charge distribution as a given source). Hence, assuming spherical symmetry we end up with an algebraic equation

$$
E+\eta^{\prime} E^{3}=E_{0}
$$

with $\eta^{\prime}=16 \pi \eta$. The solution is always in the form $\mathbf{E}\left[\mathbf{E}_{0}\right]$. One can extend the above theory to two-loops [6] in which case the algebraic equation will become a quintic polynomial equation of the form

$$
E+\eta_{1} E^{3}+\eta_{2} E^{5}=E_{0}
$$

where $\eta_{1} \simeq \eta^{\prime}$.

\section{THE ELECTRIC FIELD INSIDE THE HYDROGEN ATOM ACCORDING TO NON-LINEAR ELECTRODYNAMICS}

We can apply the results of the Euler-Heisenberg theory in the electrostatic case for $\mathbf{E}_{0}=\mathbf{E}_{p}$ where $\mathbf{E}_{p}$ is the electric field calculated using the electromagnetic form-factors. The results are shown in figure 2. As the electric field calculated from the form-factors is not a correction to the $1 / r^{2}$ Coulomb field at small distances so is the result from EulerHeisenberg not a correction to $E_{0}$ at small distances. Since the significant changes happen only at short distances, both modifications (the finite size and the light-light modifications) will induce only small corrections to the energy levels.

The energy content of the electric field is given by $\mathscr{E}[E]=\int \mathbf{E}^{2} d^{3} x$. For $E=E_{p}$ we get

$$
\mathscr{E}\left[E_{p}\right] \simeq 1 \mathrm{MeV}
$$

which, given the uncertainties in the form-factors, is close to the pair production threshold of $2 m_{e}$. On the other hand we obtain

$$
\mathscr{E}\left[E_{\gamma \gamma, 1 l o o p}\right] \simeq 0.26 \mathrm{MeV}
$$

in the one-loop Euler-Heisenberg theory and

$$
\mathscr{E}\left[E_{\gamma \gamma, 2 l o o p}\right] \simeq 0.076 \mathrm{MeV}
$$

in its two-loops version. Both results are safely below the pair production threshold. 


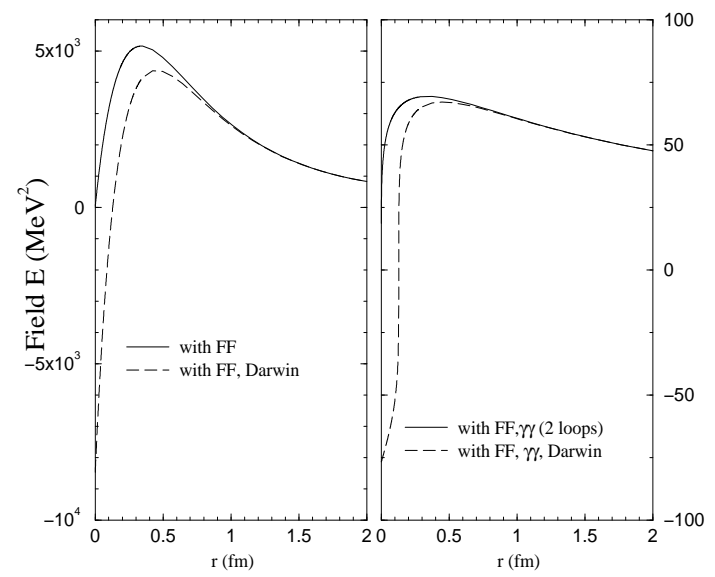

FIG. 2: Electric field inside the hydrogen atom. On the left are shown the results from form-factors (FF) with and without the proton Darwin term. On the right we show the same electric field as emerging from the Euler-Heisenberg theory.

\section{DISCUSSION}

Perturbations in the hydrogen atom can be viewed as additional contributions to the Coulomb potential suppressed by higher powers of the fine structure constant $\alpha$ (for instance the Uehling potential [7]) or as very short range modifications of the Coulomb potential. The best paradigm is given by the finite size corrections to the Coulomb part. In the short range these modifications can not be considered as perturbations of $1 / r$, but their short range character qualifies them as perturbative contributions in the hydrogen atom. A similar situation occurs when we calculate the full non-linear contributions of light-light scattering. In the short range, where the non-linear effects are important, the modifications of the EulerHeisenberg theory are not perturbative contributions to the Coulomb potential, but as in the case of the finite size effects they will contribute perturbatively to the observables of the hydrogen atom. Moreover, the electric field extracted from the electromagnetic form-factors via the Maxwellian theory is too strong as its energy content is close to the pair production threshold. In contrast to this, the electric field in the Euler-Heisenberg theory is much smaller in the short range and its energy comes out to be safely below the pair production threshold. How this affects the electron Darwin term and the hydrogen observables remains 
to be explored [8].

[1] G. Breit, Phys. Rev. 34, 553 (1929)

[2] V. B. Berestetskii, E M: Lifshitz and L. O. Pitaevskii, Quantum Electrodynamics, 2nd edition, Oxford, Butterworth-Heinemann 2007

[3] F. Garcia Daza, N. G. Kelkar and M. Nowakowski, J. Phys. G39, 035103 (2012)

[4] N. G. Kelkar, F. Garcia Daza and M. Nowakowski, Nucl. Phys. B864, 382 (2012); D. Bedoya Fierro, N.G. Kelkar and M. Nowakowski, JHEP 1509, 215 (2015), arXiv:1410.4228

[5] W. Heisenberg and H. Euler, Z. Phys. 98, 714 (1936)

[6] B. Körs and M. G. Schmidt, Eur. Phys. J. C6 (1999) 175

[7] E. A. Uehling, Phys. Rev. 48, 55 (1935)

[8] M. Nowakowski, N. G. Kelkar, D. Bedoya Fierro and A. D. Bermudez Manjarres, Static Field Configurations in the Euler-Heisenberg Theory (under preparation). 